\title{
Correlation of aspergillus skin hypersensitivity with the duration and severity of asthma
}

\author{
Vishal Chopra1, Hardik Jain1, Akhil D. Goel2, Siddharth Chopra1, Ashrafjit S. Chahal1, \\ Neha Garg1, Vidhu Mittal1 \\ 1 Department of Pulmonary Medicine, Government Medical College, Patiala \\ 2 Department of Social and Preventive Medicine, All India Institute of Medical Sciences, New Delhi, India
}

\begin{abstract}
Asthma is a significant health problem worldwide and Allergic Bronchopulmonary aspergillosis (ABPA) complicates the course of 1-2\% of patients of asthma. Aspergillus skin hypersensitivity (AH) is the first step for diagnosis of ABPA. This study was conducted to know the correlation of AH with severity and duration of asthma. Patients, age $>15$ years, of asthma attending this hospital from January 2015 to December 2015 were included. Asthma was diagnosed clinically and confirmed with spirometry. Of 282 patients 206 patients were AH positive. The AST-positivity in patients having severe asthma (96.8\%) was higher than that in patients having mild (36.8\%) and moderate asthma (80.4\%). The median (IQR) duration of asthma of AH positive patients was $5.0 \mathrm{yrs}$. This study emphasized the need of ABPA screening by intradermal skin test especially in patients having severe asthma and/or those having asthma for longer duration in order for early diagnosis of ABPA.
\end{abstract}

\section{Introduction}

Asthma is an inflammatory disease of the airways characterised by airway hyper-responsiveness and airflow limitation [1]. It is a significant public health problem with more than 300 million affected individuals worldwide [2].Aspergillus is a common cause of severe pulmonary allergic disease [3]. The most common species affecting humans are Aspergillus fumigatus, Aspergillus flavus, Aspergillus niger and Aspergillus tamari, of which Aspergillus fumigatus is responsible for about

Corresponding author: Prof. Vishal Chopra, Department of Pulmonary Medicine, Government Medical College, Sangrur Road, New Lal Bagh, Patiala 147001, India. E-mail: drvishalchopra@hotmail.com

Key words: Aspergillus skin hypersensitivity; asthma; ABPA.

Received for publication: 5 April 2017

Accepted for publication: 22 July 2017

(C) Copyright V. Chopra, et al., 2017

Tipografia PI-ME Editrice, Italy

Monaldi Archives for Chest Disease 2017; 87:826

doi: 10.4081/monaldi.2017.826

This article is distributed under the terms of the Creative Commons Attribution Noncommercial License (by-nc 4.0) which permits any noncommercial use, distribution, and reproduction in any medium, provided the original author(s) and source are credited.
95\% of Aspergillus-related illnesses in humans. These species can cause a variety of clinical manifestations ranging from hypersensitivity in Allergic Bronchopulmonary Aspergillosis (ABPA) especially in asthmatics, to an active infection in locally destructive disease, or in the most severe form as disseminated infection in immune-compromised patients [4].

ABPA is the best recognized manifestation of hypersensitivity to $A s$ pergillus antigens in patients with long standing atopic asthma. It consists of an immunologically mediated lung damage caused by hypersensitivity to the antigens of the fungus Aspergillus Fumigatus. This disease is generally believed to complicate the course of $1-2 \%$ of patients with bronchial asthma and between $2 \%$ and $15 \%$ of patients with cystic fibrosis [5].

Aspergillus skin hypersensitivity (AH) is defined by the presence of an immediate-type cutaneous hypersensitivity to A fumigatus antigens [6]. It is one of the major criteria described by Rosenberg-Patterson for the diagnosis of ABPA. This study is aimed to know the prevalence of $\mathrm{AH}$ in patients of bronchial asthma and to correlate $\mathrm{AH}$ with the duration and severity of bronchial asthma. Though the prevalence of AH in asthma has been studied earlier but as per our knowledge just a few studies have highlighted the correlation of $\mathrm{AH}$ with severity and no study has evaluated the correlation of $\mathrm{AH}$ with duration of asthma.

\section{Methods}

This study was conducted on patients of bronchial asthma presenting to the Department of Tuberculosis and Chest diseases, Government Medical College, Patiala from January 2015 to December 2015 after obtaining clearance from the Ethics Committee of our institute. Written informed consent was obtained from the patients. The patients were subjected to clinical history, physical examination, spirometry and an intradermal Aspergillus skin test (AST) with Aspergillus fumigatus antigen.

Patients were included in the study after confirming the diagnosis of bronchial asthma by identifying respiratory symptoms such as shortness of breath, cough, wheezing, or chest tightness, and demonstrating variable expiratory airflow limitation by performing spirometry [7]. The patients who had age less than 15 years, pregnancy, chronic liver disease, chronic renal failure, uncontrolled diabetes mellitus, chronic heart failure and failed to give informed consent were excluded.

Severity of asthma was assessed as per GINA guidelines of 2014 [7]. Mild asthma is one that is well controlled with Step 1 or Step 2 treatment, i.e., with as-needed reliever medication alone, or with low intensity controller treatment such as low dose ICS, leukotriene receptor antagonists or chromones. Moderate asthma is well controlled with Step 3 treatment, e.g. low dose ICS/LABA. Severe asthma is the one that requires Step 4 or Step 5 treatment, e.g. high dose ICS/LABA, to prevent it from becoming 'uncontrolled', or asthma that remains 'uncontrolled' despite this treatment. 
Aspergillus skin test was done in all the enrolled asthma patients using the intradermal technique. Aspergillus antigens belonging to the species Aspergillus fumigatus were used.

\section{Procedure}

All anti-histaminics and corticosteroids were stopped $72 \mathrm{~h}$ before testing. The flexor aspect of forearm was cleaned with soap and water and was air dried. Taking all aseptic precautions, $0.2 \mathrm{ml}$ of phosphate buffer saline was injected intradermally using a 26 -gauge needle, raising a bleb of $2 \mathrm{~mm}$. In the same way, $0.2 \mathrm{ml}$ of Aspergillus fumigatus antigen was injected next to it at a distance of $4 \mathrm{~cm}$. After 15 minutes, the site of injection was evaluated. A wheal of $3 \mathrm{~mm}$ or more was considered positive.

Aspergillus hypersensitivity (AH) was defined as the presence of immediate cutaneous hyperreactivity to the Aspergillus antigen. Commercially available standardized antigens supplied by ALCIT (India) Private Limited were used.

\section{Statistical analysis}

Qualitative variables like gender and asthma severity were described using counts and percentages and compared using Chi Square test or Fischer Exact Test as applicable. Quantitative variables like age were described using mean and standard deviation and analysed using unpaired $t$ test. Duration of asthma in years was described using median and inter-quartile range and compared using Mann Whiteney U Test. Diagnostic accuracy indicators like sensitivity, specificity, positive predictive value and negative predictive value were used to ascertain if severe asthma can by itself help in diagnosing or predicting AST status. For assessing the benefit of duration of asthma in diagnosing or predicting AST status, we performed a receiver operator characteristic (ROC) analysis. Data was entered and analysed using IBM SPSS v.20.

\section{Results}

A total of 282 asthma patients presented over the one year period from January 2015 to December 2015. Mean age was 35.7 \pm 14.1 and 147 (52.1\%) were females. Of the 282 asthma patients, 82 (29.1\%) had mild, 107 (37.9\%) had moderate and 93 (33\%) had severe asthma. The median (IQR) duration of asthma was 4.0(10.5) years. 206 (73.1\%) patients were found to be reactive for Aspergillus skin test (AST) with aspergillus fumigatus antigen.

The 206 AST reactive cases had a similar age $(\mathrm{p}=0.296)$ and gender ( $\mathrm{p}=0.363$ ) distribution as compared to the 76 AST non-reactive patients. However, AST reactive patients had significantly more cases of moderate and severe asthma while AST non-reactive cases tended to have mild asthma $(\mathrm{p}<0.001)$. Similarly, AST reactive patients had a higher median duration of asthma $(\mathrm{p}<0.001)$ as compared to AST nonreactive cases (Table 1).

Severe asthma (as compared with moderate and mild asthma) had a low sensitivity (43.7\%), high specificity (96.1\%), high positive predictive value (96.8\%) and low negative predictive value (38.6\%) for a positive AST. ROC curve analysis of duration of asthma revealed an AUC of 0.7 (95\% CI 0.63 - 0.77) indicating that duration of asthma is a fairly good predictor of the outcome of AST [Fig 1]. Optimal duration cut-off was found to be 2.25 years where highest sensitivity of $76.2 \%$ and specificity $53.9 \%$ was observed. At this cut-off, duration has a positive predictive value of $81.8 \%$ and negative predictive value of $45.6 \%$.

There was a significant correlation between severe asthma and duration of more than 2.25 years (Chi Square $=48.7, \mathrm{df}=1, \mathrm{p}<0.001$ ). To account for this, we performed a multivariate logistic regression analysis which showed that both severe asthma $(\mathrm{OR}=14.23,95 \% \mathrm{CI}: 4.23$ - 47.87) and duration of more than 2.25 years $(\mathrm{OR}=1.98,95 \% \mathrm{CI}$ : $1.10-3.56)$ were independent significant risk factors for AST positivity.

\section{Discussion}

In patients of bronchial asthma, aspergillus hypersensitivity can be considered as the first step in the development of ABPA, and ABPA can be conceptualised as an exaggerated form of Aspergillus hypersensitivity [8]. This study was conducted to determine if asthma severity and duration can affect the occurrence of aspergillus hypersensitivity in these patients.

In our study, of the 282 patients with bronchial asthma, 206 (73.0\%) showed immediate cutaneous hypersensitivity to aspergillus fumigatus antigen. The results of studies worldwide in this regard have been variable. The studies of Benatar et al. [9] and Attapattu [10] demonstrated Aspergillus hypersensitivity in $46 \%$ and $37 \%$ patients of bronchial asthma respectively. In a study conducted by Agarwal et al. [11] on 546 patients of bronchial asthma, AST was positive in $39.5 \%$ patients, whereas in another study by Agarwal et al. [12] involving 417 patients of asthma, AST was positive in $52.5 \%$ patients.

As compared to the previous studies the percentage of $\mathrm{AH}$ in patients of bronchial asthma has been found to be markedly higher in our study. This may be attributed to the fact that ours is a tertiary chest diseases hospital where most patients come with a higher severity and a longer duration of asthma. Also majority of the patients were from rural background, had agriculture as the main occupation, were rearing cattle and had biomass fuel exposure. Median duration of asthma in our patients was 4 years with the minimum duration being 0.25 years ( 3 months), and the maximum being 45 years. Of the total 282 patients, 90 had an asthma duration of 2 years or lesser, of which $49(54.44 \%)$ were positive for AST. On the other hand, the remaining 192 patients

Table 1. Comparison characteristics of AST reactors and non-reactors.

\begin{tabular}{|c|c|c|c|}
\hline & $\begin{array}{l}\text { AST Reactive } \\
(n=206)\end{array}$ & $\begin{array}{l}\text { AST Non-reactive } \\
(n=76)\end{array}$ & p value \\
\hline Age, mean \pm SD & $35.1 \pm 13.8$ & $37.1 \pm 14.9$ & $0.296 \mathrm{a}$ \\
\hline Gender, Female & $104(70.75 \%)$ & $43(29.25 \%)$ & $0.363^{b}$ \\
\hline \multicolumn{4}{|l|}{ Severity of asthma } \\
\hline Mild & $30(36.60 \%)$ & $52(63.40 \%)$ & $<0.001 \mathrm{~b}$ \\
\hline Moderate & $86(80.40 \%)$ & $21(19.60 \%)$ & \\
\hline Severe & $90(96.80 \%)$ & $03(03.20 \%)$ & \\
\hline Duration of asthma in years, median (IQR) & $5.0(11.5)$ & $2.0(4.5)$ & $<0.001 \mathrm{c}$ \\
\hline
\end{tabular}

AST, Aspergillus fumigatus Skin Test. aIndependent $t$-test; bChi Square test; cMann-Whitney U Test. Figures in parentheses indicate row-wise percentages. 
had an asthma duration of more than 2 years, of which 157 (81.77\%) were positive for AST. This result was statistically significant, implying that asthma of a longer duration showed a better correlation with positive AST as compared to asthma of a relatively shorter duration.

In the present study, asthma severity was assessed as per GINA guidelines of 2014 [7], and accordingly, the patients were categorised as having mild, moderate and severe asthma. The proportion of ASTpositivity in patients having severe asthma (96.8\%) was higher than that in patients having mild (36.8\%) and moderate asthma (80.4\%) and this result was statistically significant. This implied that patients with severe asthma are much more likely to have a positive AST than those having mild or moderate asthma. This result is concurrent with the results of earlier studies on this subject. The study conducted by Zureik et al. [13] found that the proportion of patients with sensitisation to moulds increased with increasing severity. Similarly 0'Driscoll et al. [14] in their study found that positive skin tests to all allergens were commoner in the group with severe asthma (multiple hospital admissions) than patients with milder asthma. In another study conducted by Agarwal et al. [5] in North India, the occurrence of Aspergillus hypersensitivity was significantly higher in the acute severe asthma group than in the outpatient bronchial asthma group.

However, there are certain studies which failed to find this correlation. The study by Siroux et al. [15] found that the type and intensity of allergic sensitisation to aeroallergens were not associated with any criteria of asthma severity. Similarly, the study by Agarwal et al. [12] found that the severity of asthma as per the GINA criteria was similar in the Aspergillus sensitive and the AST negative groups.

From this discussion, it is clear that in patients of bronchial asthma, Aspergillus skin hypersensitivity has a positive correlation with the duration and severity of asthma i.e., patients having more severe asthma and those with a longer duration of the disease are more likely to have aspergillus skin hypersensitivity, and thus are at an increased risk of developing ABPA. Early diagnosis of ABPA is important because timely treatment has been shown to control exacerbations, decrease relapses and prevent the development of bronchiectasis and pulmonary fibrosis

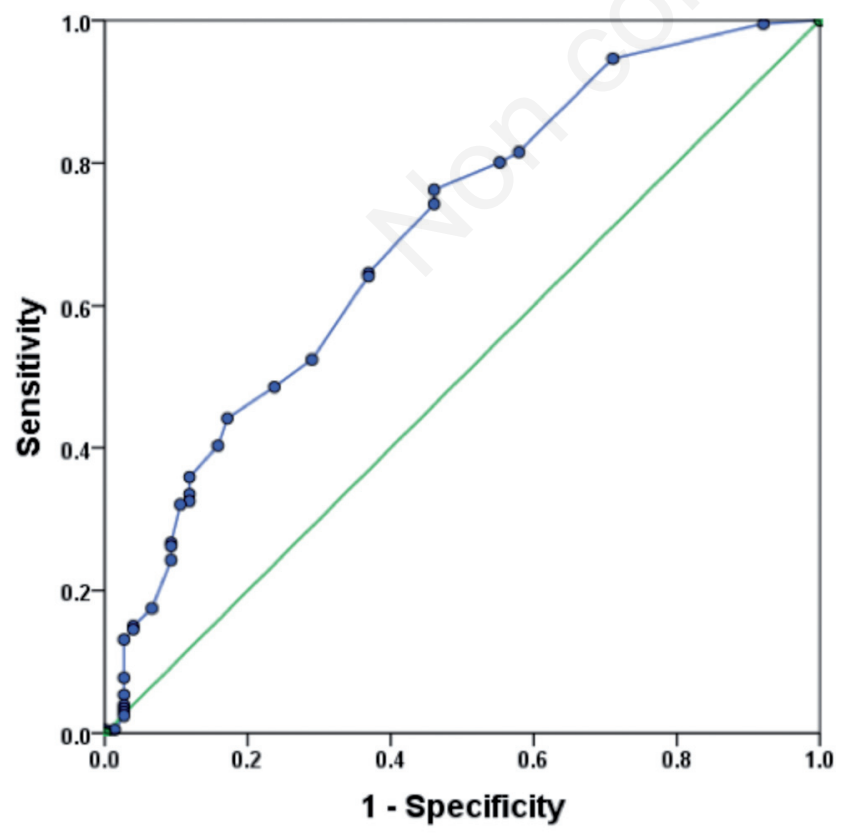

Figure 1. Receiver operator characteristic curve of duration of asthma in predicting Aspergillus fumigatus reactors.
[16]. Keeping this in mind, we suggest that these patients should be routinely screened for ABPA using an intradermal AST for timely diagnosis and initiation of proper treatment.

\section{Conclusions}

$\mathrm{AH}$ is an indicator of development of $\mathrm{ABPA}$ and according to our study it has a high rate in patients of bronchial asthma. Also, the rate of $\mathrm{AH}$ is directly proportional to the severity of asthma and its duration. This emphasizes the need for ABPA screening by an intradermal Aspergillus skin test, in all patients of asthma in general, and in patients having severe asthma and those having asthma for a longer duration in particular, for early diagnosis and treatment of ABPA, in order to prevent irreversible lung damage.

\section{References}

1. Hoogsteden HC, Verhoeven GT, Lambrecht BN, Prins JB. Airway inflammation in asthma and chronic obstructive pulmonary disease with special emphasis on the antigen-presenting dendritic cell: influence of treatment with fluticasone propionate. Clin Exp Allergy 1999;29(Suppl.2):116-24.

2. Denning DW, O'Driscoll BR, Powell G, et al. Randomized controlled trial of oral antifungal treatment for severe asthma with fungal sensitization: The Fungal Asthma Sensitization Trial (FAST) study. Am J Respir Crit Care Med 2009;179:11-8.

3. Dagenais TRT, Keller NP. Pathogenesis of Aspergillus fumigatus in Invasive Aspergillosis. Clin Microbiol Rev 2009;22:447-65.

4. Zmeili OS, Soubani AO. Pulmonary aspergillosis: a clinical update. QJM 2007;100:317-34.

5. Agarwal R, Nath A, Aggarwal AN, Gupta D, Chakrabarti A. Aspergillus hypersensitivity and allergic bronchopulmonary aspergillosis in patients with acute severe asthma in a respiratory intensive care unit in North India. Mycoses 2010;53:138-43.

6. Agarwal R. Allergic bronchopulmonary aspergillosis. Chest 2009; 135:805-26.

7. Global Initiative for Asthma. Global Strategy for Asthma Management and Prevention, 2014. Available from: www.ginasthma.org. 2014.

8. Agarwal R. Allergic Bronchopulmonary Aspergillosis: Lessons Learnt from Genetics. Indian J Chest Dis Allied Sci 2011;53:137-40.

9. Benatar SR, Keen GA, Du Toit Naude W. Aspergillus hypersensitivity in asthmatics in Cape Town. Clin Allergy 1980;10:285-91.

10. Attapattu MC. Allergic bronchopulmonary aspergillosis among asthmatics. Ceylon Med J 1991;36:45-51.

11. Agarwal R, Gupta D, Aggarwal AN, et al. Allergic bronchopulmonary aspergillosis: lessons from 126 patients attending a chest clinic in north India. Chest 2006;130:442-8.

12. Agarwal R, Noel V, Aggarwal AN, et al. Clinical significance of Aspergillus sensitisation in bronchial asthma. Mycoses 2011;54:e531-8.

13. Zureik M, Neukirch C, Leynaert B, Liard R, Bousquet J, Neukirch F. Sensitisation to airborne moulds and severity of asthma: cross sectional study from European Community respiratory health survey. BMJ 2002;325:411.4.

14. O'Driscoll BR, Hopkinson LC, Denning DW. Mold sensitization is common amongst patients with severe asthma requiring multiple hospital admissions. BMC Pulm Med 2005;5:4.

15. Siroux V, Oryszczyn MP, Paty E, et al. Relationships of allergic sensitization, total immunoglobulin E and blood eosinophils to asthma severity in children of the EGEA study. Clin Exp Allergy 2003;33:746.51.

16. Bateman ED. A new look at the natural history of aspergillus hypersensitivity in asthmatics. Respir Med 1994;88:325.7. 